\title{
Standardization of blood flow measurements by automated vascular analysis from power Doppler ultrasound scan
}

\author{
Yi Yin*a, Pádraig Looney ${ }^{\mathrm{a}}$, Sally L. Collins ${ }^{\mathrm{ab}}$ \\ aNuffield Dept. of Women's \& Reproductive Health, University of Oxford, Oxford, UK, OX3 9DU; \\ 'Fetal Medicine Unit, The Women's Centre, John Radcliffe Hospital, Oxford, UK, OX3 9DU
}

\begin{abstract}
Power Doppler ultrasound imaging provides a non-invasive method to explore tissue vascularity in real-time. It has farreaching clinical utility, for example to assess the perfusion of organs such as the placenta. In this study, a fully automated method was proposed to standardize the power Doppler signals in the placental bed to estimate the perfusion by a technique known as fractional moving blood volume (FMBV). The uterine vasculature was extracted by a region growing algorithm from 3D power Doppler scan and further localized based on the placenta segmentation obtained by a multi-class fully Convolution Neural Network (CNN). The largest vessel close to the volume of interest (VOI) in placenta was identified within which the average power Doppler signal was used as a standardization value to correct the signal attenuation in placenta bed to estimate the FMBV measurement. This is the first successful attempt to automatically identify individual blood vessel segments from a complex uterine vascular plexus. The proposed method was performed in twenty $3 \mathrm{D}$ power Doppler scans of first trimester placenta with promising results. The mean \pm STD FMBV value was $21.35 \% \pm 9.43 \%$. With further analysis and evaluation in large dataset, the proposed method will serve as an efficient tool for assessing the blood perfusion in placenta bed.
\end{abstract}

Keywords: vasculature, blood flow, deep learning, placenta, power Doppler, 3D ultrasound

\section{INTRODUCTION}

Doppler ultrasound is the primary non-invasive imaging modality performed in pregnancy to assess the blood flow within placenta and fetal organs. The power Doppler (PD) ultrasound is less affected by the angle of insonation than color Doppler and sensitive to multiple directions of flow and low flow velocities, therefore more useful to assess tissue perfusion. However, like all forms of ultrasound, PD ultrasound signal is attenuated by the tissue which it passes through. To compensate for the attenuation and permit the direct inter-patient comparison, it is necessary to standardize the power Doppler signal. The only validated method for estimating tissue perfusion from PD ultrasound is fractional moving blood volume (FMBV), which was first proposed by Rubin et al. ${ }^{1,2}$ for 2D application. Later, Stevenson et al. extended this 2D FMBV measurement into 3D and used it for in a utero placental study ${ }^{3}$. In this paper, we proposed a novel and fully automated estimation of 3D FMBV in placental bed from PD ultrasound scans of the first trimester placenta, combining deep learning and image processing techniques.

The FMBV estimation requires identification of a large reference vessel close to the target of interest which can be assumed to have $100 \%$ vascularity. This vessel is then used as a standardization point to normalize the PD signals in the target region. This can be performed manually but to be clinically useful as a screening test, the calculation of FMBV in the placental bed needs to be fully automated. Due to the signal sensitivity to noise in small vessels and the sensitivity to the motion of tissue, accurate extraction of complex vasculature using power Doppler is challenging. In most of the studies using power Doppler, blood vessels are usually segmented by thresholding ${ }^{3-5}$. However, the unavoidable noise during imaging means using just a simple thresholding method may not be robust enough. In this study, we applied a multi-seed region growing method to segment the uterine vasculature from 3D PD ultrasound scans, which performed better in keeping the vessel connectedness than simple thresholding.

*yi.yin@wrh.ox.ac.uk; phone +44 (0)1865 572259

Copyright 2020 Society of Photo-Optical Instrumentation Engineers (SPIE). One print or electronic copy may be made for personal use only. Systematic reproduction and distribution, duplication of any material in this publication for a fee or for commercial purposes, and modification of the contents of the publication are prohibited.

Citation:

Yi Yin, Pádraig Looney, Sally L. Collins, "Standardization of blood flow measurements by automated vascular analysis from power Doppler ultrasound scan," Proc. SPIE 11314, Medical Imaging 2020: Computer-Aided Diagnosis, 113144C (16 March 2020); https://doi.org/10.1117/12.2549349 
Our target of interest, the placenta bed, is located at the utero-placental interface (UPI) where the maternal blood enters the placenta. The UPI is detected based on the placenta volume segmentation which might be estimated by semi-automatic tools, e.g. the commercial tool VOCAL ${ }^{\mathrm{TM}}$ and Random Walker method ${ }^{6}$, or automated deep learning tools ${ }^{7,8}$. We applied a fully convolutional neural network $(\mathrm{CNN})$ to the corresponding B-mode ultrasound volumes which segmented the placenta, amniotic fluid and fetus using a multi-class model with state-of-the-art accuracy. This provides a fast and accurate localization of the UPI. The reference vessel used for standardizing the PD signals in the placenta bed was picked automatically from the segmented uterine vasculature distributed at a certain distance from the UPI into the uterus.

In this paper, we proposed a fully automated method for the estimation of tissue perfusion measurement FMBV. We demonstrated its application in the human placenta by using the 3D power Doppler ultrasound scans of the first trimester placenta. Promising results were obtained from early pregnancy patient data showing the potential of our method to serve as a screening testing tool.

\section{METHOD DESCRIPTION}

The 3D ultrasound data used in this study was collected as part of a research study conducted with full local ethical approval ${ }^{9}$. The scans were acquired in the first-trimester of pregnancy by a GE Voluson E8 scanner and a 3D/4D curvedarray abdominal transducer. 143 patients underwent scanning between 11 weeks and 13 weeks 6 days' gestation. 141 data were available in this study. A coordinate transformation was performed to convert the raw data saved in Kretz file format from toroidal system to Cartesian system for further processing ${ }^{10}$. After coordinate transformation, the proposed automated method was applied to the data which consists of vasculature analysis, placental segmentation and 3D FMBV estimation by standardization of the power Doppler signal.

\subsection{Vasculature analysis}

The whole vasculature was extracted from 3D PD ultrasound scans by region growing algorithm initiating from multi-seed points (Fig. 1). The seed points were selected in local brightest region close to the placenta volume estimated by a CNN which will be discussed in Section 2.2. This allowed the region growing method to detect all of the blood vessel segments, even if they were disconnected for some unexpected reasons, e.g. being cut at the border of the image volume. The algorithm used the confidence connected criterion to compute the mean and standard deviation of signal intensity values of initial seed regions and included the neighbor voxels with intensity values falling inside the range around the mean. The intensity range was updated by these new included voxels and new neighbor voxels were evaluated to see whether their intensity values fell within the new range. The algorithm iterated until no new voxels were added in the updated region. The algorithm was implemented by using the filters of ITK $^{11}$.

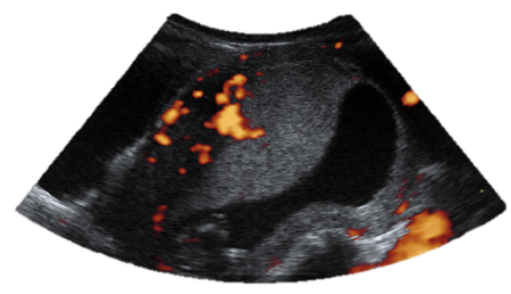

(a) Power Doppler signal

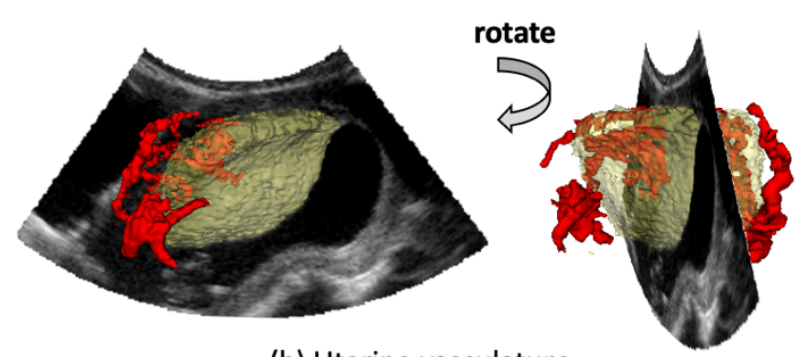

(b) Uterine vasculature

Figure 1. Uterine vasculature segmentation from 3D power Doppler scans: (a) power Doppler signal overlaid on a 2D section of the B-mode ultrasound scans; (b) blood vessels detected surrounding the placenta (yellow).

Next, the vessel skeleton was extracted by a 3D medial axis based thinning method $^{12}$, using the binary vessel segmentation as input image. The skeleton of one voxel width represented the geometrical central line of the vascular tree (Fig. 2(a)). Based on the estimated skeleton, the vasculature was separated into segments by using watershed-based algorithm. The skeleton image was filtered with an all-ones kernel of size $3 \times 3 \times 3$ voxels. The bifurcated and intersected points of the 
centerline had values greater than 2 in the filtered image. By removing these bifurcated and intersected points, the vessel skeleton was separated into segments. The separated skeleton segments were treated as markers overlaid to the binary vessel segmentation, from which the watershed algorithm flooded basins. The vascular volume was then split into segments as illustrated in Fig. 2(b).

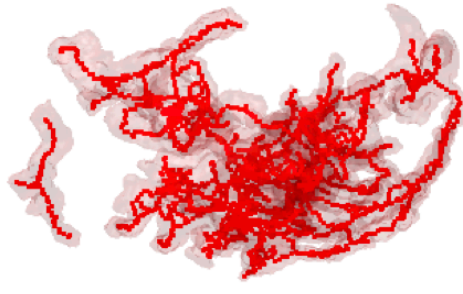

(a) Vascular centerline

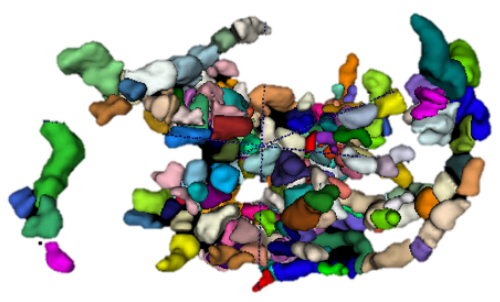

(b) Separated vessel segments

Figure 2. Vasculature skeletonization and separation: (a) skeleton of one voxel width represented the vessel centerline; (b) vessel segments split from the uterine vasculature segmentation.

When blood vessels lie very close to each other, no obvious border can be observed and they might be segmented as an individual vessel. The watershed-based separation procedure took the geometrical centerlines as markers. This succeeded in splitting the bifurcated and intersected structures, but not in splitting the tangled structure of close vessels. If there was more than one vessel in close proximity, multiple intensity peaks were seen in the vessel cross-section, an intersection between the vessel volume and a plane perpendicular to the local vessel centerline. Based on this observation, the real individual blood vessels showing single peak in the cross-sections are identified from these vessel segments to be considered as candidates of a reference vessel used for FMBV estimation.

\subsection{Placental segmentation}

A fully convolutional neural network $(\mathrm{CNN})$ was applied to segment the placenta as well as amniotic fluid and fetus from the 3D B-mode volume. 2,393 patient data were involved in training and validation of the model to find the optimal parameters. The 2,393 placentas were labeled and validated by three experts, which was described in details in previous work $^{7} .300$ cases were selected randomly and further labeled the amniotic fluid and fetus. This CNN architecture using two pathways combing the placenta prediction of single-class model and the amniotic fluid and fetus predictions of the multi-class model. The single class pathway had a similar architecture to the U-net as shown in Fig. 3. Transfer learning technique was adopted to initialize the single-class pathway by using the optimized parameters of this model trained on 2,093 labelled placental volumes. The multi-class pathway had a similar architecture but had four output classes in the last layer before Softmax function. The CNN was trained for 40 epochs with a batch size of 30, by 300 cases with placenta, amniotic fluid and fetus segmented.

The model obtained the best performance was applied to the power Doppler data for predictions. Once the segmentation of the placenta, amniotic fluid and fetus was obtained. The most important structure UPI was defined as the interface between the placental volume and a total volume combining the placenta, amniotic fluid and fetus. The target of interest and the reference vessel were both identified based on the accurate localization of UPI. 


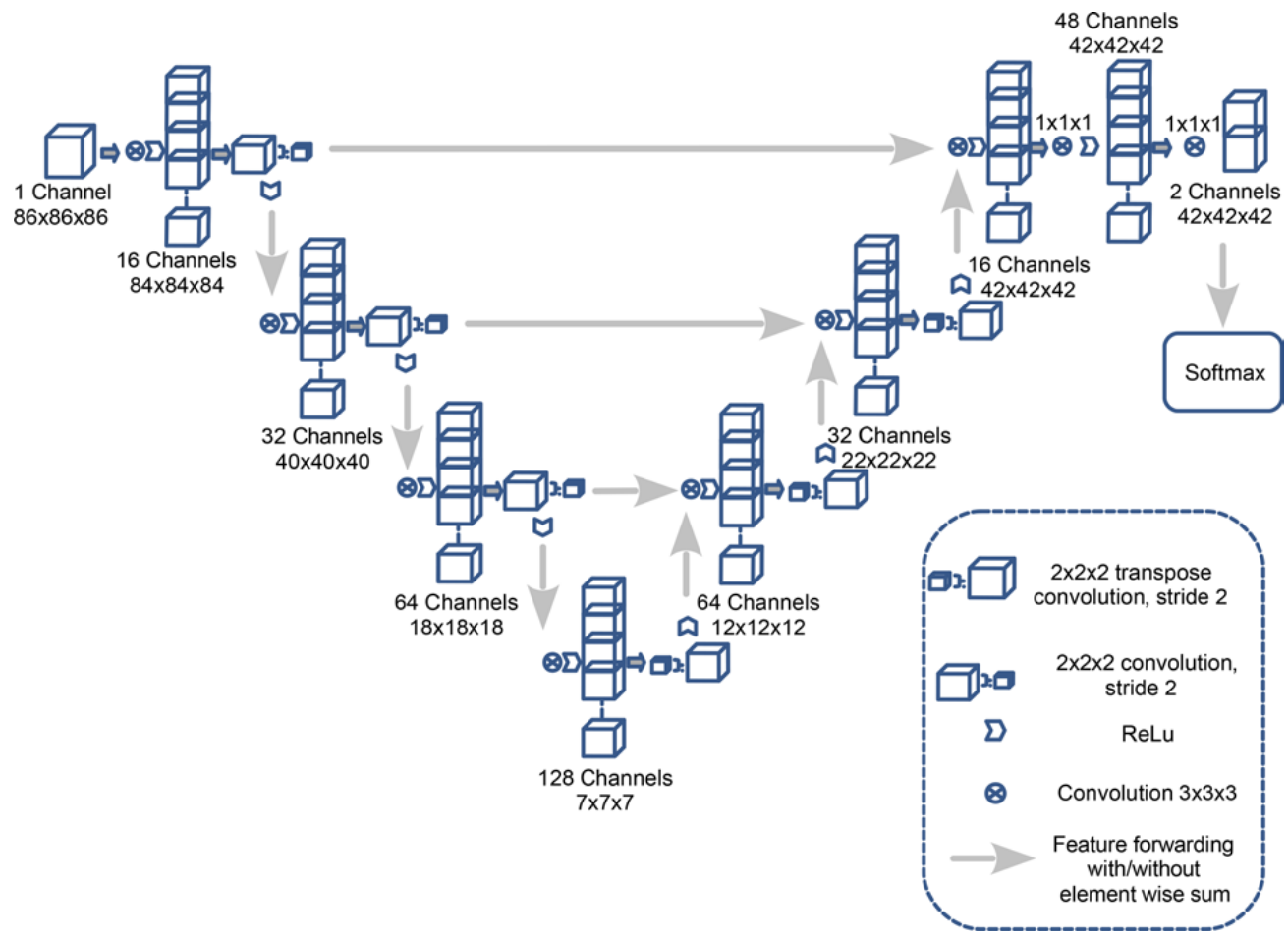

Figure 3. Architecture of the single class fully convolutional neural network (From Looney et al. ${ }^{7}$ )

\subsection{FMBV estimation}

For a given patient ultrasound scan, the uterine vasculature was segmented from the power Doppler data and the UPI (yellow surface in Fig. 4(a)) was extracted based on the multi-class prediction from the corresponded B-mode data. The largest individual vessel distributed within $1 \mathrm{~cm}$ from the UPI into the uterine was identified as the reference vessel (cyan segment marked by a white circle in Fig. 4(a)). The power Doppler signal intensities of the reference vessel were used to build a cumulative distribution curve as shown in Fig. 4(b). The knee value of the cumulative curve was found according to the method proposed by Rubin et al. ${ }^{3}$ used as a standardization value.

The target VOI within the placenta $(0.5 \mathrm{~cm}$ from the UPI into the placenta shown in transparent blue in Fig. 4(a)) was normalized by comparing the voxel intensities with the standardization value (Equation 1). The resulting FMBV value was the mean of the normalized power Doppler signal values expressed as a percentage related to $100 \%$ vascularity (Equation 2).

$$
\begin{array}{r}
I_{t}(X)= \begin{cases}I(X), & \text { if } I(X)<I_{s v} \\
I_{s v}, & \text { if } I(X) \geq I_{s v}\end{cases} \\
F M B V=\frac{1}{N} \sum_{X=1}^{N} \frac{I_{t}(X)}{I_{s v}}
\end{array}
$$

Here, $I_{s v}$ is the standardization value estimated from the reference vessel. $I(X)$ is the signal intensity of a voxel within the target region. $I_{t}$ is the truncated signal intensity and $N$ is the number of voxels within the target volume. 


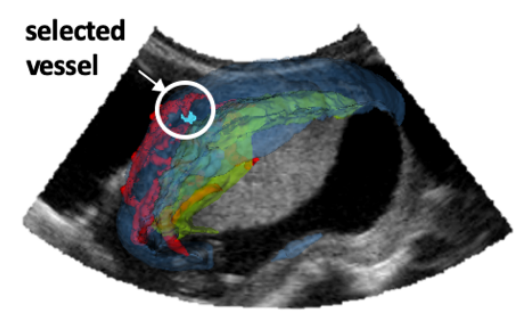

(a) Reference vessel

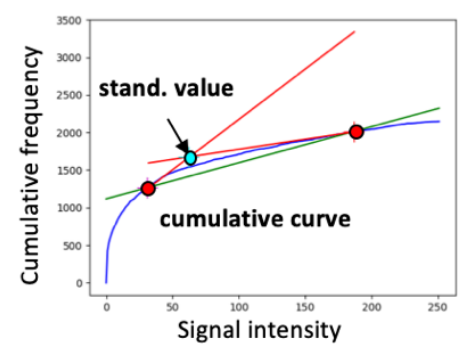

(b) Standardization value

Figure 4. The fractional moving blood volume (FMBV) estimation: (a) reference vessel selection; (b) estimation of standardization value from the signal intensity cumulative distribution.

\section{RESULTS}

The proposed method was applied to 20 patient data of first trimester pregnancy, which were a subset of the available 141 power Doppler ultrasound scans. These cartesian data were transformed from a toroidal coordinated system and resampled with a spatial resolution of $0.6 \mathrm{~mm}$. The signal intensity ranged between 0 to 255 . The regions of high power Doppler signal intensity greater than 200 were detected whose center points were considered to be the seed points to initiate the multi-seed region growing algorithm to segment uterine vasculature. The appearance was consistent with what was subjectively judged to be representative of the vascular plexus by an experienced sonographer (Fig. 5).
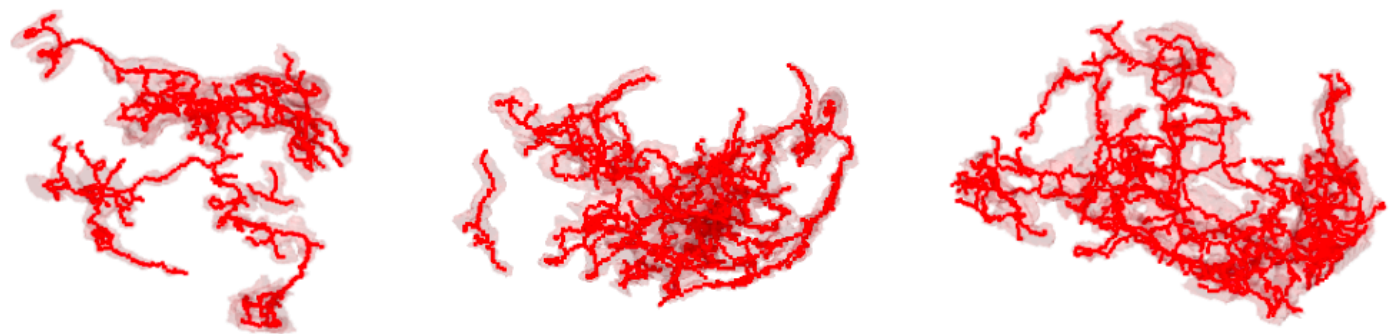

Figure 5. Examples of extracted vasculature (light pink volume) and vessel centerline (red).

The multi-class fully convolutional neural network obtained a Dice similarity coefficient (DSC) of 0.81 for placenta segmentation on 141 patient data. DSC is defined as in Equation 3, where $A$ and $B$ are two segmentations. This state-ofthe-art performance of segmentation permitted the accurate localization of the UPI, based on which to identify the target placental volume and the reference blood vessel being use as the standardization point for estimation of FMBV.

$$
D S C=\frac{2|A \cap B|}{|A|+|B|}
$$

The standardization value derived from the 20 patient data was $140 \pm 51$ (mean \pm STD). The estimated 3D FMBV was $21.35 \% \pm 9.43 \%$ (mean \pm STD), which fell within the range of FMBV values of placenta bed presented by Stevenson et al. ${ }^{3}$.

\section{CONCLUSION}

In this study, a fully automated method was introduced to standardize the power Doppler signals to estimate tissue perfusion measurement FMBV. Promising results were obtained from the 3D power Doppler scans. To the best of our knowledge, we managed for the first time to automatically identify the reference individual blood vessel segment from a 
complex vascular plexus as the 'standardization' point for estimation of tissue perfusion with FMBV. The ultrasound artefacts induced by tissue motion (e.g. 'flash' artefacts) or cardiac cycle might affect the signal intensities resulting in false segmentation of the blood vessels. Denoising of the images should be developed in the further study as a preprocessing step for accurate vasculature extraction. The CNN provided state-of-the-art segmentation of uterus structures for standardization and target localization. Including training data from different sources in the future might improve the accuracy as data diversity between manufacturers might have influence on the performance of the neural network. The automated procedure which has the potential to revolutionize the ability of ultrasound to produce vascular measurement not only within the placental bed but also in other organs and tumors.

\section{ACKNOWLEDGEMENTS}

The authors would like to thank Gordon N. Stevenson, Kypros H. Nicolaides, Walter Plasencia, Malid Molloholli, and Stavros Natsis for providing the data and their contributions to the previous project. The authors and the research reported in this paper were supported by the Eunice Kennedy Shriver National Institute of Child Health and Human Development (NICHD) Human Placenta Project of the National Institutes of Health under award number UO1-HD087209.

\section{REFERENCES}

[1] Rubin, J. M., Adler, R. S., Fowlkes, J. B., Spratt, S., Pallister, J. E., Chen, J. F. and Carson, P. L., "Fractional moving blood volume: estimation with power Doppler US," Radiology, 197(1), 183-190 (1995)

[2] Rubin, J. M., Bude, R. O., Fowlkes, J. B., Spratt, R. S., Carson, P. L. and Adler, R. S., "Normalizing fractional moving blood volume estimates with power Doppler US: defining a stable intravascular point with the cumulative power distribution function," Radiology, 205(3), 757-765 (1997)

[3] Stevenson, G. N., Collins, S. L., Welsh, A. W., Impey, L. W. and Noble, J. A., "A technique for the estimation of fractional moving blood volume by using three-dimensional power Doppler US," Radiology, 274(1), 230-237 (2015)

[4] Huang, Y. H., Chen, J. H., Chang, Y. C., Huang, C. S., Moon, W. K., Kuo, W. J., Lai, K. J. and Chang, R. F., "Diagnosis of solid breast tumors using vessel analysis in three-dimensional power Doppler ultrasound images," Journal of digital imaging, 26(4), 731-739 (2013)

[5] Shia, W. C., Chen, D. R., Huang, Y. L., Wu, H. K. and Kuo, S. J., "Effectiveness of evaluating tumor vascularization using 3D power Doppler ultrasound with high-definition flow technology in the prediction of the response to neoadjuvant chemotherapy for T2 breast cancer: a preliminary report," Phys Med Biol., 60(19), 7763 7778 (2015)

[6] Stevenson, G. N., Collins, S. L., Ding, J., Impey, L. and Noble, J. A., "3-D Ultrasound Segmentation of the Placenta Using the Random Walker Algorithm: Reliability and Agreement," Ultrasound in Medicine and Biology, 41(12), 3182-3193 (2015)

[7] Looney, P., Stevenson, G. N., Nicolaides, K. H., Plasencia, W., Molloholli, M., Natsis, S. and Collins, S. L., "Fully automated, real-time 3D ultrasound segmentation to estimate first trimester placental volume using deep learning," JCI Insight, 3(11), pii: 120178 (2018)

[8] Yang, X., Yu, L., Li, S., Wen, H., Luo, D., Bian, C., Qin, J., Ni, D., and Heng, P. A., "Towards automated semantic segmentation in prenatal volumetric ultrasound," IEEE Trans Med Imaging, 38(1), 180-193 (2019)

[9] Collins, S.L., Stevenson, G. N., Noble, J. A., Impey, L. and Welsh, A.W., "Influence of power Doppler gain setting on Virtual Organ Computer-aided AnaLysis indices in vivo: can use of the individual sub-noise gain level optimize information?" Ultrasound Obstet. Gynecol., 40(1), 75-80 (2012)

[10]Looney, P. T., Stevenson, G. N. and Collins, S. L., "3D ultrasound file reading and coordinate transformations," Journal of Open Source Software, 4(33), 1063 (2019)

[11] Johnson, H. J., McCormick, M., Ibáñez, L., and Consortium, T. I. S., "The ITK Software Guide, 3rd ed., Kitware", Inc., In press. [Online]. Available: http://www.itk.org/ItkSoftwareGuide.pdf, (2013)

[12]Lee, T. C., Kashyap, R. L., Chu, C. N., "Building Skeleton Models via 3-D Medial Surface/Axis Thinning Algorithms, " CVGIP: Graphical Models and Image Processing, 56(6), 462-478 (1994) 